\title{
Mid-peripheral corneal steepening after orthokeratology
}

\author{
Mhamed Ouzzani, MSc, \\ PhD Candidate \\ University of Oran1, \\ Optometry group, LPCMME
}

\section{Catherine Wright, OD} University of Waterloo, School of Optometry and Vision Science

Luigina Sorbara, OD, MSc, FAAO, FBCLA, Diplo.

University of Waterloo, School of Optometry and Vision Science

\begin{abstract}
PURPOSE

The purpose of this study was to retrospectively examine data from patients who had undergone orthokeratology (OK) to quantify the amount of mid-peripheral steepening in tangential curvature (MP-TC) and the change in total refractive power (TRP).
\end{abstract}

\section{METHODS}

Charts were reviewed and Pentacam data were collected prior to OK and after the final visit. Variables recorded included central flattening (C-TC), the e-value, the mid-peripheral tangential curvatures (MP-TC), total corneal refractive power (TRP), the initial Rx (sphere) and initial corneal curvature (flat K). Patients were further subdivided into high and low myopia groups for comparison. All participants were fitted with Paragon CRT lenses. Data were analysed to determine the effects of lenses on TC and TRP.

\section{RESULTS}

A total of 40 patients ( 80 eyes), age $13.95 \pm 6.80 \mathrm{y}, 34$ male and 46 female, were successfully fitted with CRT lenses. The average sphere was $-4.23 \pm 0.90 \mathrm{D}$ for the high myopia group and $-1.89 \pm 0.62 \mathrm{D}$ for the low myopia group. The changes in TC and TRP from baseline were significant (both $\mathrm{P}<0.0001$ ). The difference between the amount of C-TC and the maximum area of MPTC was +/- 4.00D in the horizontal meridian and $+/-3.00$ in the vertical meridian. The amount of MP-TC change from baseline was +/- 2.00D in both meridians. There was a similar change in TRP: the distance from the centre to mid-periphery was +/- 2.50D. For C-TC, C-TRP, MP-TC, and MP-TRP there was no significant difference between the 2 groups, overall $(\mathrm{P}=0.541$ (TC) and $\mathrm{P}=0.321(\mathrm{TRP})$ ).

\section{CONCLUSION}

These results should provide valuable insights into the topographic and refractive changes that occur with orthokeratology.

KEY WORDS:

Orthokeratology, tangential curvature, total refractive power, mid-peripheral steepening

\section{INTRODUCTION}

Researchers, clinicians and manufacturers have shown growing interest in the recent dramatic increase in the prevalence of myopia. Myopia is one of the most common types of refractive error; however, it may significantly affect one's quality of life when left uncorrected or in cases where it exceeds $>-6.00 D .{ }^{1}$ Since extreme myopia (>-6.00D) can lead to lenticular and irreversible retinal changes, minimizing the amount of myopia to safer levels should be the practitioner's goal. ${ }^{2}$

A current challenge is how to manage the progression of myopia in children (MC) who are at risk of being fast progressors, that is, those with both myopic parents and siblings. Options for MC include optical methods such as multifocal contact lenses, ${ }^{3,4}$ orthokeratology $(\mathrm{OK})^{5}$ and pharmaceutical tools such as atropine. ${ }^{6}$ Increasing the time spent outdoors has also been shown to slow the progression of myopia., 
Except for the pharmaceutical method (atropine), MC tools are based on either changing the corneal shape to produce a mid-peripheral "plus lens" retinal defocus or simply adding plus to the mid-periphery of a soft contact lens. Both of these methods result in thickening of the mid-peripheral choroid, which is thought to reduce elongation of the axial length, and thus slow the progression of myopia. ${ }^{9}, 10$ With orthokeratology in particular, the curvature of the cornea is flattened centrally to correct the refractive error and steepened in the mid-periphery to create myopic defocus. ${ }^{11}$

While $\mathrm{OK}$ has been advocated as an effective method for treating MC, the fitting of OK lenses is time-consuming. Therefore, if a soft multifocal (SCL MF) lens design could achieve the same result, most practitioners would prefer this simpler fitting modality. SCL MF have a nominal $+2.00 \mathrm{D}$ add and have shown promise in patients with MC. The amount of myopic defocus induced by OK has not been well established. It appears to be related to the contact lens design, the asymmetry the lens may induce, and the baseline amount of myopia. ${ }^{12,13}$

The purpose of this study was to retrospectively examine data from patients who had undergone OK for the correction of myopia and the slowing of myopic progression (in younger patients) to quantify the amount of mid-peripheral curvature steepening and the changes in total refractive power after OK.

\section{METHODS}

The main objective of this study was to conduct a retrospective chart review in 40 participants to investigate the amount of mid-peripheral curvature steepening that occurs with OK. This study also investigated whether the amount of mid-peripheral steepening depends on the amount of central flattening, the e-value, the initial Rx (sphere), or the initial corneal curvature (flat K). Participants who were patients of the Contact Lens Clinic and who had provided their signed consent for chart review over the past four years were included in the study.

This analysis entailed measurement of the initial corneal/ocular characteristics versus the final changes in curvature and Rx, which represented the final successful fitting visit. Variables included central flattening, the e-value, the mid-peripheral tangential curvature, total corneal refractive power, the initial $\mathrm{Rx}$ (sphere) and initial corneal curvature (flat $\mathrm{K})$. The group was further subdivided into high (>-3.25D, $\mathrm{n}=40$ eyes) and low myopia $(<-3.00 \mathrm{D}, \mathrm{n}=40$ eyes) for comparison.

Data were collected and exported from the "4 map selectable" option on a Pentacam". The Oculus Pentacam" (Oculus, Wetzlar, Germany) is a standard optometric class II medical device and was used for the measurement of tangential corneal curvature (TC) and total refractive corneal power (TRP). The Pentacam measures geometrical height (elevation) values in $\mathrm{mm}$ of both the anterior and posterior corneal surfaces, which are subsequently converted into values of axial (sagittal) or tangential curvature, as shown in Fig. 1.

Figure 1: Tangential front curvature map

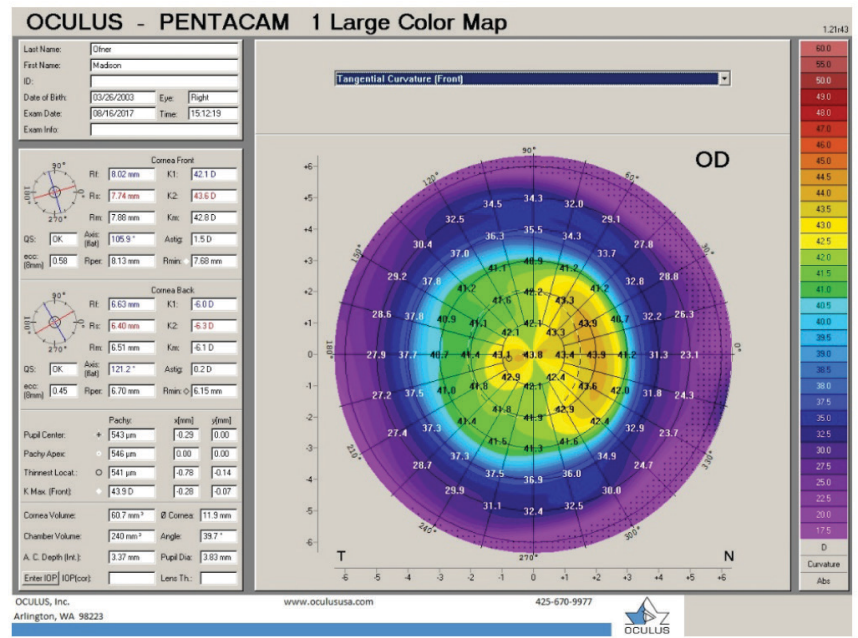


On the other hand, the total refractive power map (Fig. 2) uses only values from the anterior surface. It calculates corneal power according to Snell's law of refraction, assuming a refractive index of 1.3375 to convert curvature into refractive power (Fig. 3). ${ }^{14}$ This is a map that other Placido topographers, along with tomographers such as the Pentacam, may show because only the anterior surface needs to be considered in the calculation of refractive power.

Figure 2: Total refractive power map

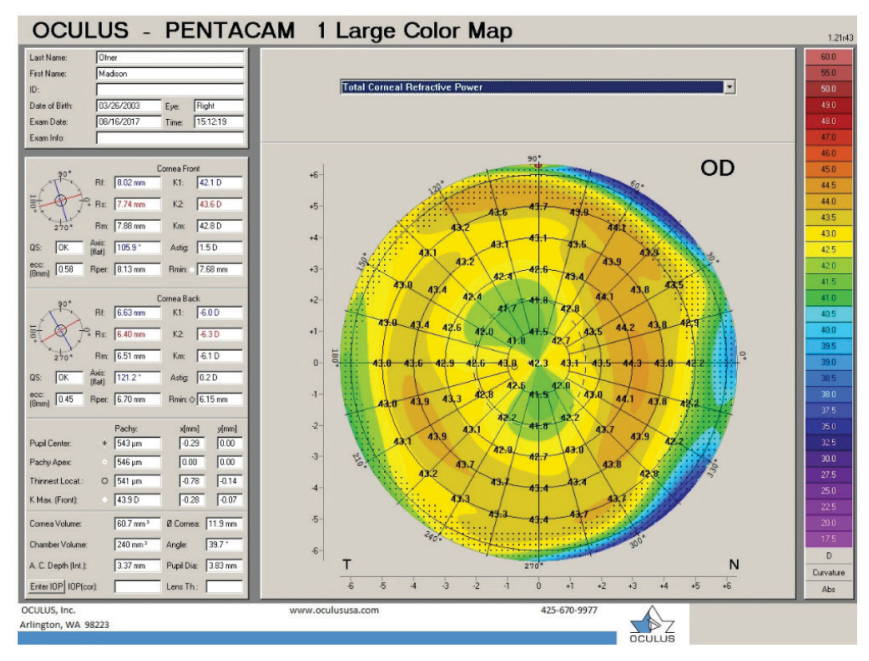

Figure 3: Snell's law of refraction

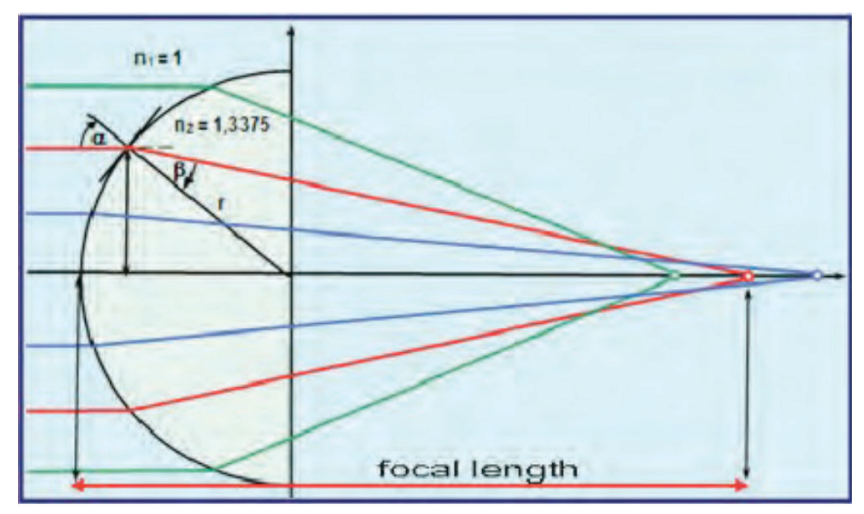

The data were analyzed both at baseline and at the final visit. The data were collected centrally and at mid-peripheral locations (nasal and temporal, superior and inferior in $0.5 \mathrm{~mm}$ increments). Refractive data at baseline and at the final visit were collected from the electronic medical record.

The contact lenses selected were Paragon CRT ${ }^{\circ}$ lenses (Paragon, Mesa, AZ) made of HDS 100 (a 100 DK fluorosilicone acrylate material), for the correction of myopia and to reduce the progression of myopia..$^{15}$ The lenses are designed to have congruent anterior and posterior surfaces, each consisting of three zones: 1) the central spherical zone, 2) a mathematically designed sigmoidal corneal proximity "Return Zone", and 3) a non-curving "Landing Zone". The lens design also includes a convex elliptical edge terminus that smoothly joins the anterior and posterior surfaces (CRT ${ }^{ø}$ fitting guide, Paragon Vision Sciences).

A successful fit was confirmed for all participants, on the basis of a manifest refraction, best-corrected visual acuity, slit lamp observations with grading of findings, unaided visual acuity and a centred appearance of corneal flattening and mid-peripheral steepening as shown on the post-orthokeratology topographic maps. 
Descriptive statistics were gathered on baseline variables (age, gender, refractive error distribution, etc.). Data analysis was conducted using STATISTICA (Tulsa, AZ). Parametric statistics (repeated measures ANOVA and t-tests) were used to analyse the effects of lenses on refractive error, corneal topography and topographic total corneal refractive power.

RESULTS

A total of 40 patients ( 80 eyes) with a mean age of $13.95 \pm 6.80$ (range 7.5 to $45.25 \mathrm{y}$ ), 34 males and 46 females, were successfully fitted with CRT lenses. The overall mean sphere value was $-3.06 \pm 1.41 \mathrm{D}$, the mean e-value was $0.46 \pm$ 0.10 (from 0.11 to 0.76 ), and the $\mathrm{K}$ readings were $42.51 \pm 0.23$ and $43.44 \pm 0.26$, in the horizontal and vertical meridians, respectively, measured with the Pentacam.

We subdivided the participants according to their spherical refractive error, as the cylindrical component was negligible, with 40 eyes in each group. There was no significant difference in age between the two groups (Group 1 (high myopia): $14.56 \pm 7.12 \mathrm{y}$ and Group 2 (low myopia): $12.56 \pm 4.27 \mathrm{y}, \mathrm{P}>0.05$ ). Group 1 had a mean sphere of $-4.23 \pm$ $0.90 \mathrm{D}$ (ranging from -3.25 to $-6.00 \mathrm{D}$ ), a mean e-value of $0.47 \pm 0.10$ (0.26 to 0.76 ), and mean K readings of $42.98 \pm 1.12$ and 44.07 $\pm 1.47 \mathrm{D}$ in the horizontal and vertical meridians, respectively. Group 2 had a mean sphere of $-1.89 \pm 0.62 \mathrm{D}$ $(-0.75$ to $-3.00 \mathrm{D})$, a mean e-value of $0.45 \pm 0.10$ (0.11 to 0.58$)$, and mean K readings of $42.36 \pm 1.15 \mathrm{D}$ horizontally and $43.26 \pm 1.25 \mathrm{D}$ vertically. There was no difference in e-values between the 2 groups $(\mathrm{P}>0.05)$, despite the differences in refractive sphere $(\mathrm{P}<0.0001)$, or in the $\mathrm{K}$ readings when comparing both the horizontal and vertical meridians $(\mathrm{P}=0.03$ and $\mathrm{P}=0.02$, respectively).

Examples of the TRP and TC maps at the final visit (after orthokeratology) are shown in Fig. 4.

Figure 4: Post-Orthokeratology examples of tangential and refractive power maps
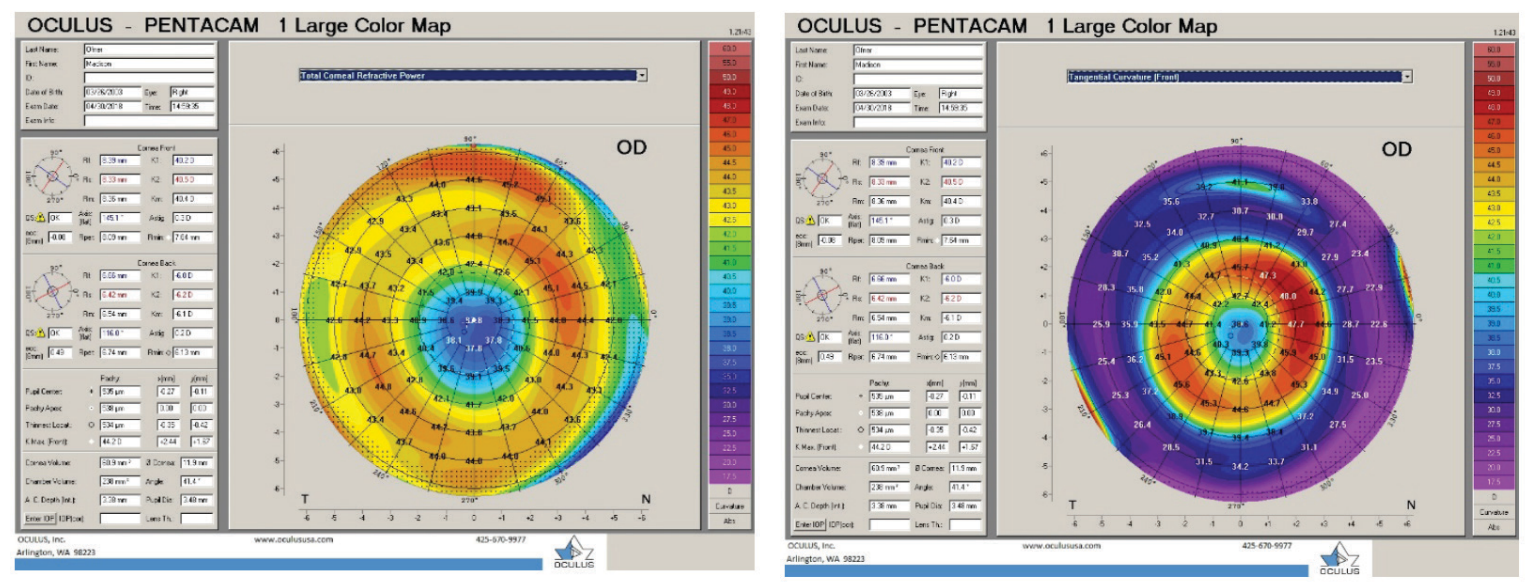

Figure 5a shows the change in tangential topographic curvature (TC) for all participants at $0.5 \mathrm{~mm}$ steps from the centre, both nasally $(\mathrm{N})$ and temporally $(\mathrm{T})$, from baseline to after OK treatment; there was a significant change $(\mathrm{P}<0.0001)$ in the horizontal meridian. The difference between the amount of central flattening $(\mathrm{C}-\mathrm{TC})$ and the maximum area of mid-peripheral steepening (MP-TC) was +/- 4.00D. On the other hand, the amount of mid-peripheral change from baseline was +/- 2.00D. There is some asymmetry; the nasal portion is steepened more significantly than the temporal region, indicating more nasal decentration $(\mathrm{p}=0.0001)$.

The vertical MP-TC and MP-TRP were not as intensively analyzed as the horizontal meridian values because the upper eyelid often prevented the successful collection of data on the superior and inferior cornea, and thus the determination of the exact value of mid-peripheral steepening. In general, though, the vertical results were similar to the horizontal results in that the amount of maximal mid-peripheral steepening (MP-TC) was $+/-3.00 \mathrm{D}$ and the amount of mid-peripheral change from baseline was again +/- 2.00D ( $<<0.0001)$, compared to baseline (Fig. 5b). It also showed asymmetry, in that the inferior portion was steepened significantly more than the superior portion $(\mathrm{p}=0.003)$. 
Figure 5a: Central flattening and mid-peripheral steepening in the horizontal meridian after orthokeratology in the total group $(n=40)$. Vertical bars denote $95 \%$ confidence intervals. Figure $5 \mathrm{~b}$ : Central flattening and mid-peripheral steepening in the vertical meridian after orthokeratology in the total group $(n=40)$. Vertical bars denote $95 \%$ confidence intervals.

5a)

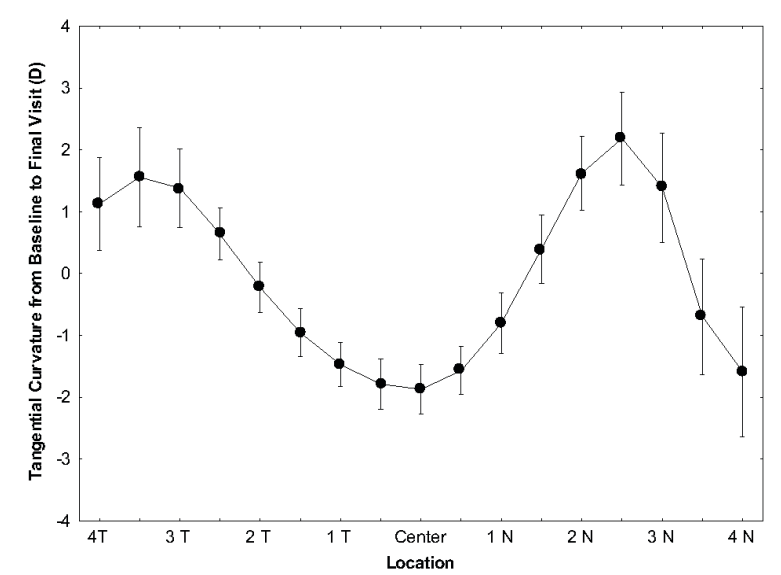

5b)

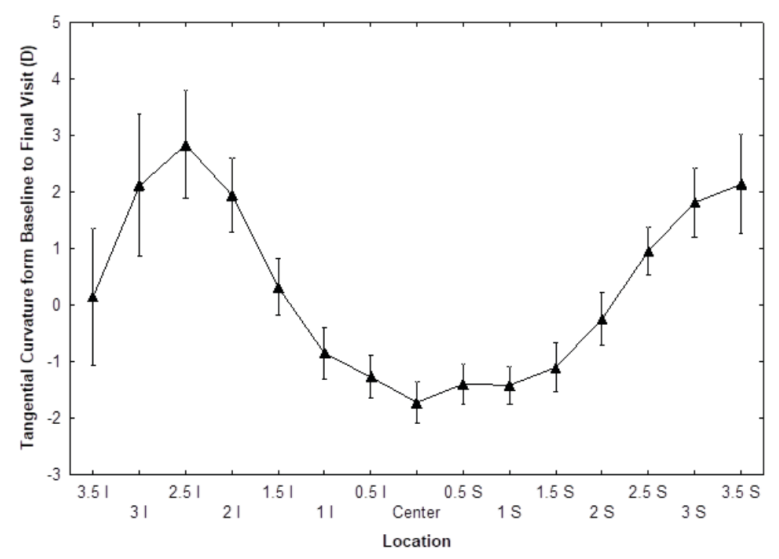

The changes in tangential curvature measured at $0.5 \mathrm{~mm}$ steps from the centre both nasally $(\mathrm{N})$ and temporally $(\mathrm{T})$, comparing the high (Group 1) versus low (Group 2) myopia groups, from baseline, are shown in Fig. 6. Centrally and in the mid-periphery, there was a marginally significant difference between the 2 groups by location $(\mathrm{P}=0.049)$. On the other hand, there was a significantly different amount of central flattening (C-TC) and temporal steepening (MP-TC) (post-hoc, $\mathrm{P}=0.01$ ) where Group 2 showed more asymmetry, compared to baseline.

Figure 6: Central flattening and mid-peripheral steepening after orthokeratology in the high and low myopia groups ( $n=20$ for each group). Vertical bars denote $95 \%$ confidence intervals.

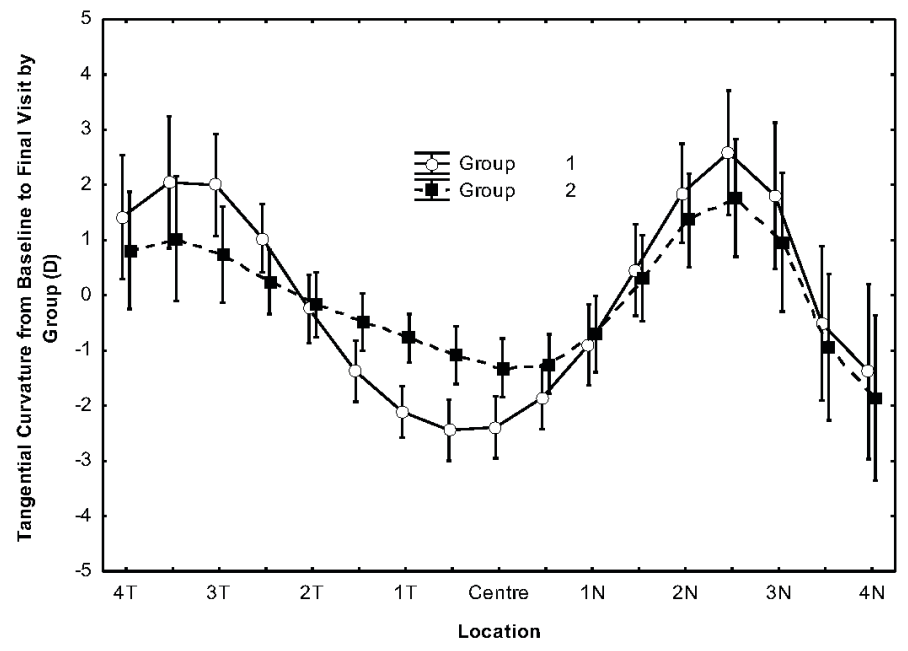

Figure 7a shows the changes in the total corneal refractive power (TRP) for all participants, measured at $0.5 \mathrm{~mm}$ steps from the centre both nasally (N) and temporally (T) after OK. Note that the total refractive power (defined as the Snell's Law refractive map which takes into account the eye's spherical aberration and considers both the front and back corneal surfaces) ${ }^{16}$ decreases centrally (+/- 2.00D) and increases in the mid-periphery (+/- 0.75D) significantly $(\mathrm{P}<0.0001)$ from baseline to the final visit. The inferior-superior comparisons (Fig. $7 \mathrm{~b})$ were also similar to the horizontal nasal and temporal findings in that the inferior cornea had a higher refractive power difference $(0.88 \pm 1.80 \mathrm{D})$ than the superior cornea $(0.17 \pm 1.06 \mathrm{D})$ when compared to baseline, likely due to lens inferior decentration $(\mathrm{P}<0.0001)$. 
Figure 7a: Changes in the total corneal refractive power after orthokeratology in the horizontal meridian in the total group $(n=40)$. Vertical bars denote $95 \%$ confidence intervals. Figure $7 \mathrm{~b}$ : Changes in the total corneal refractive power after orthokeratology in the vertical meridian in the total group $(n=40)$. Vertical bars denote $95 \%$ confidence intervals.

7a)

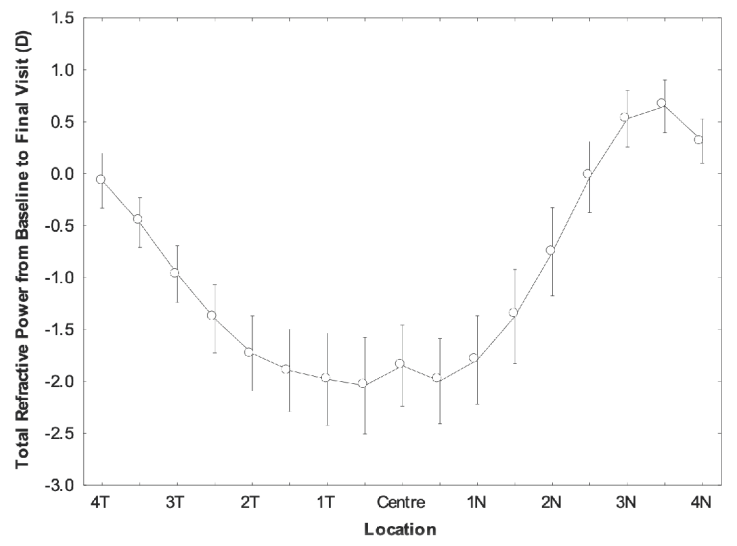

7b)

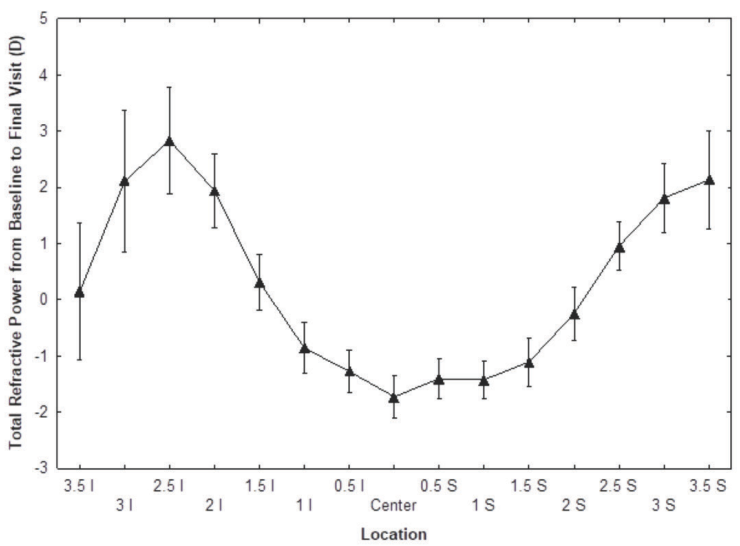

When comparing groups 1 and 2 there were no significant differences $(\mathrm{P}=0.321)$ in the total refractive power at each location measured at $0.5 \mathrm{~mm}$ steps from the centre, both nasally $(\mathrm{N})$ and temporally $(\mathrm{T})$, as shown in Fig. 8 . In addition, there were no differences when comparing nasal, central and temporal TRP (post-hoc, $\mathrm{P}>0.05$ ). On the other hand, significant differences were seen when comparing centre to mid-periphery for both groups $(\mathrm{P}<0.0001)$.

Figure 8: Changes in the total corneal refractive power after orthokeratology in the high and low myopia groups ( $n=20$ for each group). Vertical bars denote $95 \%$ confidence intervals.

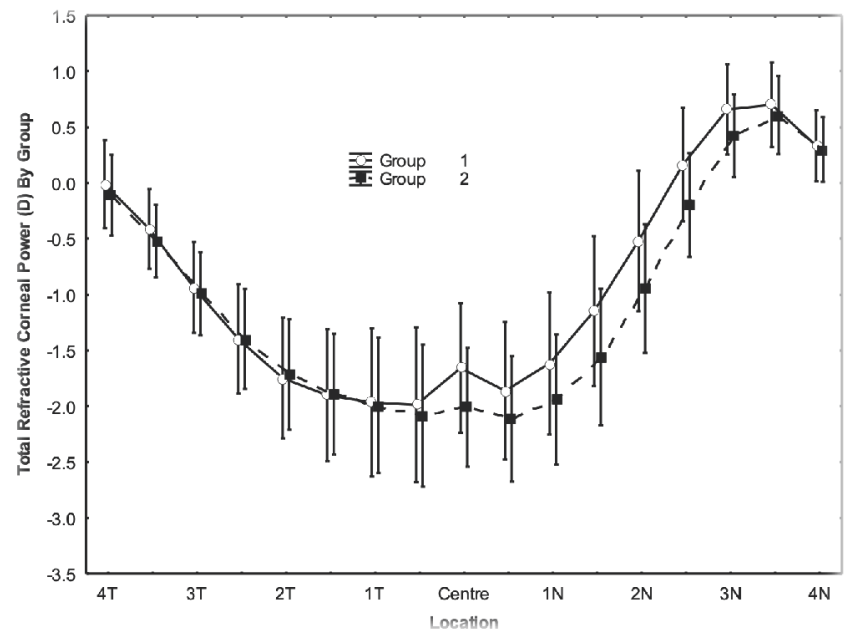

The changes in topographic and total refractive data from the centre to mid-periphery after OK (at final visit only) were examined for each group, and the results are shown in Fig. 9. There were significant differences in topographic curvature and total refractive power $(\mathrm{P}<0.0001)$ when the two groups were compared by location $(\mathrm{P}<0.0001)$. 
Figure 9: Changes in topographic curvature and total refractive data from the centre to mid-periphery after OK in the high and low myopia groups ( $n=20$ for each group). Vertical bars denote $95 \%$ confidence intervals.
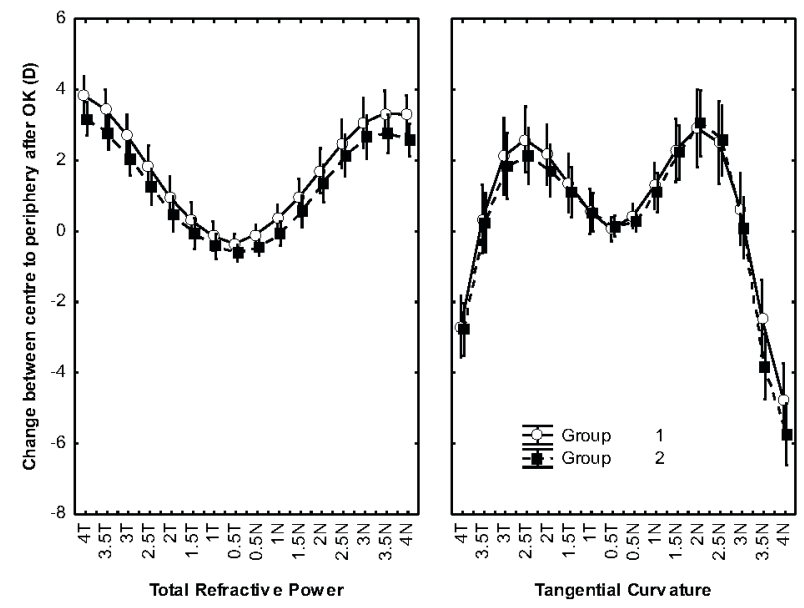

When we compared the central changes to the mid-peripheral changes after OK at the final visit only, the amount of mid-peripheral change was asymmetric, as seen in Fig. 10, where the tangential curvature was affected by decentration more nasally than temporally $(\mathrm{P}<0.0001)$. The change in total refractive power was also significantly affected nasally, but to a lesser degree $(\mathrm{P}<0.0001)$.

Figure 10: Change between the centre and periphery, both nasally and temporally, for the tangential curvature and total corneal refractive power, highlighting the asymmetry of the results of treatment $(n=40)$. Vertical bars denote $95 \%$ confidence intervals.
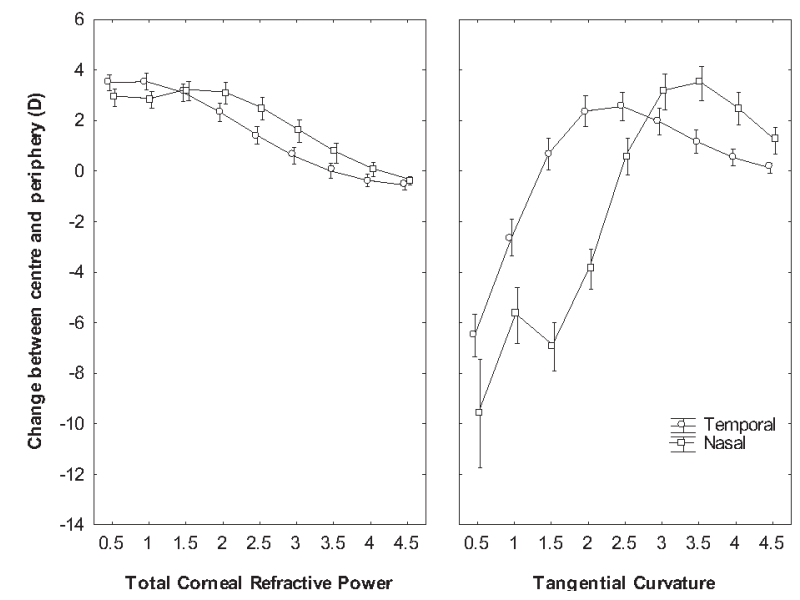

Calculations were performed to identify which predictor variables were correlated with the amount of mid-peripheral steepening. None of the predictor variables (e-value, horizontal K, and initial sphere) was significantly correlated $(\mathrm{P}>0.05)$ with the amount of mid-peripheral steepening. There were significant correlations between the initial spherical refraction and both the central tangential curvature change and the central total refractive power change $\left(\mathrm{R}^{2}=0.33\right.$ and 0.36 , respectively, $\left.\mathrm{P}<0.0001\right)$. 
DISCUSSION

Early animal and human studies indicated that a change in peripheral refractive power can reduce the progression of myopia by inducing myopic defocus on the peripheral retina. ${ }^{9,17}$ The refractive power of the eye is not constant across the entire cornea. In fact, the greatest refractive power occurs peripherally due to spherical aberration, despite the prolate elliptical (flattening from the centre to the periphery) shape of the cornea. In addition, light striking the mid-periphery of the cornea does so obliquely, inducing more aberrations. ${ }^{18}$ Studies have shown that myopic eyes exhibit mid-peripheral hyperopic defocus, which results in an increase in myopia. When this is corrected using myopic defocus in the mid-periphery of the cornea, the progression of myopia may be slowed, as mid-peripheral changes dominate over any foveal changes. ${ }^{19-21}$ The CRT $^{\circledR}$ lenses in this study were designed to correct myopia. Currently, there are modifications to this reverse geometry lens design that may be more effective for controlling myopia. The purpose of this file review was to examine the topographic changes induced by this reverse lens geometry, as this is the basis of myopia control designs.

OK contact lenses create mid-peripheral myopic defocus in the para-foveal region of the retina by their unique back-surface geometry. The mid-peripheral steeper radius zone (also known as the reverse curve) starts at the edge of the back optic zone, and is the most important area in myopia control because it creates myopic defocus in the para-foveal regions. ${ }^{910}$ Many studies have shown that use of this type of contact lens is effective for managing low to moderate amounts of myopia. ${ }^{22,23}$ This study showed more specific results regarding topographical changes with $\mathrm{OK}$. Neither the amount of central flattening with corresponding mid-peripheral steepening nor the amount of total refractive power change were significantly different between the high and low myopia groups. However, central flattening and mid-peripheral steepening were found to be significantly different for TC, but not for total refractive power. This study implies that higher myopes may have greater mid-peripheral steepening compared to lower myopes in the nasal region only, as a recent study has shown, due to lens decentration with higher amounts of central flattening. ${ }^{12}$ There were no significant differences in either the K readings or the e-values between the two groups. If the significant difference between the high and low myopia groups for TC is considered, then the changes in the amounts of central and mid-peripheral steepening (average of C-TC+/-4D and MP-TC+/-2D) are significantly related to the initial refractive error. On the other hand, globally, there were no differences between the two groups for TC or TRP. There are two possible explanations that may aid in interpretation of the results. First, the topographic, and hence the related refractive, changes are due to the contact lens design. The lens design has a mid-peripheral zone steeper than the base curve by a fairly constant amount (from 500 to 550 microns). ${ }^{15}$ Therefore, it can be inferred that the amount of mid-peripheral steepening corresponds solely to the reverse geometry of the lens when the results are considered globally. Second, there is evidence that the epithelium thickens by a constant amount (between 13 and 16 microns $)^{24,25}$ with the use of OK lenses in the reverse zone, which also supports the result of having an approximately constant amount of mid-peripheral change. This is further supported by the fact that our predictor variables did not correlate with the amount of mid-peripheral steepening, as the latter did not vary with the change in refractive power.

Corneal refractive therapy and the resulting remodelling of corneal shape are controlled by mathematical laws. Munnerlyn's formula ${ }^{26}$ states that changes in the corneal shape cannot exceed the predicted amount of central flattening. In his analysis of the changes in corneal shape, Mountford ${ }^{27}$ found that refractive error induced by accelerated OK showed a mean reduction in myopia of $2.19 \pm 0.80 \mathrm{D}$, with a maximum reduction of $5.00 \mathrm{D}$, which corresponds to our results. Mountford also found correlations between apical corneal power and radius with a change in refraction. Similarly, this study found significant correlations of changes in central tangential curvature and central total refractive power with change in spherical refraction.Other studies have found a similar amount of mid-peripheral steepening, further supporting our hypothesis. ${ }^{13,28,29}$

As expected, this study showed significant changes from the centre to mid-periphery in the tangential curvature and the total corneal refractive power. This was reported by Swarbrick et al., ${ }^{30}$ who discussed the changes in topography from the centre to 3 locations nasally and temporally, from $0.5 \mathrm{~mm}$ to 1.75 and $0.25 \mathrm{~mm}$ to 2.50 , respectively and did not find a significant change in topography by location. However, our topographic data were analysed in $0.5 \mathrm{~mm}$ steps in the horizontal meridian and there were significant changes from baseline to the final visit by location. This method allowed us to determine more subtle changes, which help explain the statistical differences that were not found in Swarbrick's study. 
Studies show that the corneal remodelling that occurs with OK is a result of changes in the epithelial thickness. ${ }^{24-26,31}$ In a study by Reinstein et al., ${ }^{31}$ the mid-peripheral epithelium thickened by up to $16 \mu \mathrm{m}$ with OK. Wang et al. ${ }^{25}$ and Haque et al. ${ }^{24}$ showed that the changes in epithelial thickness, centrally and in the mid-periphery, are the same with varying amounts of initial myopia. They showed that the change in central epithelial thickness was $5.1 \% \pm 4.5 \%$ thinner than baseline and in the mid-periphery there was significant thickening (1.9\% on the temporal location and $2.4 \%$ nasally). This asymmetric amount of mid-peripheral change in corneal epithelial thickness temporally and nasally was also observed by Alharbi et al., ${ }^{26}$ who explained this phenomena in terms of slight decentration of the lenses in most cases. Asymmetric changes were also observed in this study that compared the nasal and temporal cornea with regard to TC and TRP, which could be related to the epithelial changes that were noted by others.

This study has some limitations, as it was a retrospective chart review rather than a controlled clinical trial. In addition, the sample size could be larger due to the high prevalence of myopia.

\section{CONCLUSION}

With OK lenses, the changes in mid-peripheral power and tangential topographical curvature were $+/-0.75 \mathrm{D}$ and $+/-2.00 \mathrm{D}$ respectively in the horizontal and vertical meridians. Centrally and nasally only, they varied significantly with the initial spherical refractive error. The results of this study should provide valuable insights into the topographic and refractive changes occurring with $\mathrm{OK}$ and this lens design. $\bullet$

\section{REFERENCES}

1. Rose K, Harper R, Tromans C, et al. Quality of life in myopia. Br J Ophthalmol 2000 Sep; 84(9): 1031-4.

2. Saw SM, Gazzard G, Shih-Yen EC, Chua WH. Myopia and associated pathological complications. Ophthalmic Physiol Opt 2005 Sep;25(5):381-91.

3. Aller TA, Liu M, Wildsoet CF. Myopia control with bifocal contact lenses: a randomized clinical trial. Optom Vis Sci 2016 Apr;93(4):344-52.

4. Walline JJ, Greiner KL, McVey ME, Jones-Jordan LA. Multifocal contact lens myopia control. Optom Vis Sci 2013 Nov;90(11):1207-14.

5. Sun Y, Xu F, Zhang T, et al. Orthokeratology to control myopia progression: a meta-analysis. PLoS One 2015 Apr 9;10(4):e0124535.

6. Li SM, Wu SS, Kang MT, et al. Atropine slows myopia progression more in Asian than white children by meta-analysis. Optom Vis Sci 2014 Mar;91(3):342-50.

7. Sherwin JC, Hewitt AW, Coroneo MT, Kearns LS, Griffiths LR, Mackey DA. The association between time spent outdoors and myopia using a novel biomarker of outdoor light exposure. Invest Ophthalmol Vis Sci 2012 Jul 1;53(8):4363-70.

8. Sherwin JC, Reacher MH, Keogh RH, Khawaja AP, Mackey DA, Foster PJ. The association between time spent outdoors and myopia in children and adolescents: a systematic review and meta-analysis. Ophthalmology 2012 Oct;119(10):2141-51.

9. Smith EL 3rd, Kee CS, Ramamirtham R, Qiao-Grider Y, Hung LF. Peripheral vision can influence eye growth and refractive development in infant monkeys. Invest Ophthalmol Vis Sci 2005 Nov;46(11):3965-72.

10. Smith EL 3rd, Hung LF. The role of optical defocus in regulating refractive development in infant monkeys. Vision Res 1999 Apr;39(8):1415-35.je

11. Woo GC, Chow E, Cheng D, Woo S. A study of the central and peripheral refractive power of the cornea with orthokeratology treatment. Int Contact Lens Clin 1994;21:132-6.

12. Marcotte-Collard R, Simard P, Michaud L. Analysis of two orthokeratology lens designs and comparison of their optical effects on the cornea. Eye Contact Lens 2018 Sep;44(5):322-9.

13. Maseedupally V, Gifford P, Lum E, Swarbrick H. Central and paracentral corneal curvature changes during orthokeratology. Optom Vis Sci 2013 Nov;90(11):1249-58.

14. Hr P. OCULUS Pentacam ${ }^{\circledR}$ Pentacam ${ }^{\circledR}$ HR Interpretation Guide 3rd edition. https://www.pentacam.com/fileadmin/user_upload/ pentacam.de/downloads/interpretations-leitfaden/interpretation_guideline_3rd_edition_0915.pdf, Accessed February 10, 2019.

15. Paragon CRT. Perspectives on Fitting Paragon CRT ${ }^{\circledast}$ Dual Axis ${ }^{\mathrm{TM}}$. https://www.artoptical.com/storage/docs/CRT_Dual_Axis.pdf.

16. Sanders DR, Koch DD, eds. An atlas of corneal topography. Thorofare, NJ: Slack Incorporated, 1993.
17. Sankaridurg P, Holden B, Smith E 3rd, et al. Decrease in rate of myopia progression with a contact lens designed to reduce relative peripheral hyperopia: one-year results. Invest Ophthalmol Vis Sci 2011 Dec 9;52(13):9362-7.

18. Calossi A. Corneal asphericity and spherical aberration. J Refract Surg 2007 May;23(5):505-14.

19. Atchison DA, Pritchard N, Schmid KL. Peripheral refraction along the horizontal and vertical visual fields in myopia. Vision Res 2006 Apr;46(8-9):1450-8.

20. Chen X, Sankaridurg P, Donovan L, et al. Characteristics of peripheral refractive errors of myopic and non-myopic Chinese eyes. Vision Res 2010 Jan;50(1):31-5.

21. Mutti DO, Sholtz RI, Friedman NE, Zadnik K. Peripheral refraction and ocular shape in children. Invest Ophthalmol Vis Sci 2000 Apr;41(5):1022-30.

22. Cho P, Cheung SW, Edwards M. The longitudinal orthokeratology research in children (LORIC) in Hong Kong: a pilot study on refractive changes and myopic control. Curr Eye Res 2005 Jan;30(1):71-80.

23. Walline JJ, Rah MJ, Jones LA. The children's overnight orthokeratology investigation (COOKI) pilot study. Optom Vis Sci 2004 Jun;81(6):407-13.

24. Haque S, Fonn D, Simpson T, Jones L. Corneal and epithelial thickness changes after 4 weeks of overnight corneal refractive therapy lens wear, measured with optical coherence tomography. Eye Contact Lens 2004 Oct;30(4):189-93; discussion 205-6.

25. Wang J, Fonn D, Simpson TL, Sorbara L, Kort R, Jones L. Topographical thickness of the epithelium and total cornea after overnight wear of reverse-geometry rigid contact lenses for myopia reduction. Invest Ophthalmol Vis Sci 2003 Nov;44(11):4742-6.

26. Alharbi A, Swarbrick HA. The effects of overnight orthokeratology lens wear on corneal thickness. Invest Ophthalmol Vis Sci 2003 Jun;44(6):2518-23.

27. Mountford, J. An analysis of the changes in corneal shape and refractive error induced by accelerated orthokeratology. Int Contact Lens Clin 1997;24:128-44.

28. Charman WN, Mountford J, Atchison DA, Markwell EL. Peripheral refraction in orthokeratology patients. Optom Vis Sci 2006 Sep;83(9):641-8.

29. Queirós A, González-Méijome JM, Jorge J, Villa-Collar C, Gutiérrez AR. Peripheral refraction in myopic patients after orthokeratology. Optom Vis Sci 2010 May;87(5):323-9.

30. Swarbrick HA, Wong G, O'Leary DJ. Corneal response to orthokeratology. Optom Vis Sci 1998 Nov;75(11):791-9.

31. Reinstein DZ, Gobbe M, Archer TJ, Couch D, Bloom B. Epithelial, stromal, and corneal pachymetry changes during orthokeratology. Optom Vis Sci 2009 Aug;86(8):E1006-14. 